\title{
DEFORMATION THEORIES CONTROLLED BY HOCHSCHILD COHOMOLOGIES
}

\author{
SAMUEL CAROLUS, SAMUEL A. HOKAMP, AND JACOB LAUBACHER
}

\begin{abstract}
We explore how the higher order Hochschild cohomology controls a deformation theory when the simplicial set models the 3 -sphere. Besides generalizing to the $d$-sphere for any $d \geq 1$, we also investigate a deformation theory corresponding to the tertiary Hochschild cohomology, which naturally reduces to those studied for the secondary and usual Hochschild cohomologies under certain conditions.
\end{abstract}

\section{INTRODUCTION}

Higher order Hochschild (co)homology was implicitly defined by Anderson in [1], and was given an explicit description in [7]. The case for when the simplicial set models the $d$-sphere was investigated more extensively in [5]. A deformation theory for the algebra $A[[t]]$ controlled by the higher order Hochschild cohomology over the 2-sphere was studied in [3]. One of the goals of this paper is to generalize their argument.

In Section 3 we use the simplicial structure for the 3 -sphere presented in [2], and also use their natural extension when considering the $d$-sphere for any $d \geq 1$. We show how the higher order Hochschild cohomology over the $d$-sphere controls a deformation theory, giving special attention to the case when $d=3$.

When the simplicial set models $S^{1}$, it is well known that one recovers the usual Hochschild cohomology, which was introduced in 1945 in [6]. Almost twenty years later in [4], Gerstenhaber used this Hochschild cohomology, denoted $\mathrm{H}^{*}(A, A)$, to describe deformations of the algebra $A[[t]]$. That is, for a multiplication law $m_{t}: A[[t]] \otimes A[[t]] \longrightarrow A[[t]]$ determined by $m_{t}(a \otimes b)=a b+c_{1}(a \otimes b) t+c_{2}(a \otimes b) t^{2}+\cdots$ with $\mathbb{k}$-linear maps $c_{i}: A \otimes A \longrightarrow A$, one sees that $m_{t}$ is associative $\bmod t^{2}$ if and only if $c_{1}$ is a 2-cocycle. As is classical, the class of $c_{1}$ is determined by the isomorphism class of $m_{t}$. Finally, assuming associativity $\bmod t^{n+1}$, the obstruction for associativity $\bmod t^{n+2}$ is an element in $\mathrm{H}^{3}(A, A)$.

In 2016, Staic showed in [8] that when one wants to study deformations of $A[[t]]$ that have a nontrivial $B$-algebra structure, one can use the secondary Hochschild cohomology. This cohomology theory has the property that when one takes $B=\mathbb{k}$, one recovers the usual Hochschild cohomology.

In Section 4 we study deformations of $A[[t]]$ that have nontrivial $B$-algebra and $C$-algebra structures. This is done using the tertiary Hochschild cohomology, which was introduced in [2]. This tertiary Hochschild cohomology depends on a morphism of commutative $\mathbb{k}_{\mathbf{k}}$ algebras $\theta: C \longrightarrow B$. This morphism induces a $B$-algebra and $C$-algebra structure on $A$ by way of the morphisms $\varepsilon: B \longrightarrow A$ and $\varepsilon \circ \theta: C \longrightarrow A$, respectively. We show that this is equivalent to having a family of products satisfying a generalized associativity condition.

Date: August 7, 2019.

2010 Mathematics Subject Classification. Primary 16S80; Secondary 16E40.

Key words and phrases. Deformations of algebras, higher order Hochschild cohomology, tertiary Hochschild cohomology. 
In particular, when one takes $C=\mathbb{k}$, one recovers exactly the result in [8]. Also, as a natural extension, we discuss deformations of $A[[t]]$ with $n$ nontrivial algebra structures for any $n \geq 1$.

\section{Preliminaries}

Fix $\mathbb{k}_{k}$ to be a field and denote $\otimes:=\otimes_{\mathbb{k}}$ and $\operatorname{Hom}(-,-):=\operatorname{Hom}_{\mathbb{k}}(-,-)$. Furthermore, we set $A$ to be an associative $\mathbb{k}$-algebra with multiplicative unit.

For $d \geq 1$, we begin by recalling the chain complex associated to the higher order Hochschild cohomology of the commutative $\mathbb{k}$-algebra $A$ with coefficients in the $A$-symmetric $A$-bimodule $M$ over the $d$-sphere $S^{d}$. We denote the complex

$$
0 \rightarrow M \stackrel{\delta_{0}}{\longrightarrow} \ldots \stackrel{\delta_{d-2}}{\longrightarrow} M \stackrel{\delta_{d-1}}{\longrightarrow} \operatorname{Hom}(A, M) \stackrel{\delta_{d}}{\longrightarrow} \operatorname{Hom}\left(A^{\otimes d+1}, M\right) \stackrel{\delta_{d+1}}{\longrightarrow} \ldots
$$

by $\mathbf{C}_{S^{d}}(A, M)$. It will be of particular interest when one takes $M=A$. Moreover, we focus on the map $\delta_{d}: \operatorname{Hom}(A, M) \longrightarrow \operatorname{Hom}\left(A^{\otimes d+1}, M\right)$. For any $\mathbb{k}$-linear map $f: A \longrightarrow M$, we have that

$$
\begin{aligned}
\delta_{d}(f)\left(a_{1} \otimes \cdots \otimes a_{d+1}\right)= & a_{1} \cdots a_{d} f\left(a_{d+1}\right) \\
& +\sum_{i=1}^{d}(-1)^{i} a_{1} \cdots a_{d-i} f\left(a_{d+1-i} a_{d+2-i}\right) a_{d+3-i} \cdots a_{d+1} \\
& +(-1)^{d+1} f\left(a_{1}\right) a_{2} \cdots a_{d+1} .
\end{aligned}
$$

Definition 2.1. ([1],[7]) The cohomology of the complex $\mathbf{C}_{S^{d}}(A, M)$ is called the higher order Hochschild cohomology of $A$ with coefficients in $M$ over the $d$-sphere, which is denoted by $\mathrm{H}_{S^{d}}^{*}(A, M)$.

We note that when taking $M=A$, then for any $d \geq 1$, the maps in low dimension are straightforward. Indeed for $0 \leq n \leq d-1$, we have that

$$
\delta_{n}= \begin{cases}0 & \text { if } n \text { is even } \\ \text { id } & \text { if } n \text { is odd }\end{cases}
$$

As consequence of (2.3):

Example 2.2. When $M=A$, we have the following for any $d \geq 1$ :

(i) $\mathrm{H}_{S^{d}}^{0}(A, A)=A$,

(ii) $\mathrm{H}_{S^{d}}^{n}(A, A)=0$ for all $1 \leq n \leq d-1$,

(iii) $\mathrm{H}_{S^{d}}^{d}(A, A) \cong \operatorname{Ker}\left(\delta_{d}\right)$ for when $d$ is odd, and

(iv) $\mathrm{H}_{S^{d}}^{d}(A, M) \cong \frac{\operatorname{Ker}\left(\delta_{d}\right)}{A}$ for when $d$ is even.

Next, we recall the tertiary Hochschild cohomology of a $\mathbb{k}$-algebra $A$. This algebra $A$ need not be commutative, unlike the case for the higher order Hochschild cohomology. The tertiary Hochschild homology was introduced in [2], and the cohomology is an easy adaptation, as they mentioned. For the purposes of this paper, it suffices to only consider the complex in low dimension.

Definition 2.3. ([2]) We call $(A, B, C, \varepsilon, \theta)$ a quintuple if

(i) $A$ is a $\mathbb{k}$-algebra,

(ii) $B$ is a commutative $\mathbb{k}$-algebra,

(iii) $\varepsilon: B \longrightarrow A$ is a morphism of $\mathbb{k}$-algebras such that $\varepsilon(B) \subseteq \mathcal{Z}(A)$, 
(iv) $C$ is a commutative $\mathbb{k}$-algebra, and

(v) $\theta: C \longrightarrow B$ is a morphism of $\mathbb{k}$-algebras.

We next consider a quintuple $\mathcal{Q}=(A, B, C, \varepsilon, \theta)$, and we let $M$ be an $A$-bimodule which is $B$-symmetric (and therefore $C$-symmetric). We denote the complex

$0 \rightarrow M \stackrel{\beth^{0}}{\longrightarrow} \operatorname{Hom}(A, M) \stackrel{\beth^{1}}{\longrightarrow} \operatorname{Hom}\left(A^{\otimes 2} \otimes B \otimes C, M\right) \stackrel{\beth^{2}}{\longrightarrow} \operatorname{Hom}\left(A^{\otimes 3} \otimes B^{\otimes 3} \otimes C^{\otimes 4}, M\right) \stackrel{\beth^{3}}{\longrightarrow} \ldots$

by $\mathbf{C}^{\bullet}(\mathcal{Q} ; M)$. Again, it will be of particular interest when one takes $M=A$. First, however, we describe these maps in low dimension. As noted in [2], one can arrange these elements to form a tetrahedron. Since working with an element expressed in three dimensions is laborious, we instead follow the arrangement in [2] and consider a two-dimensional sliced representation. For ease of notation, we will consider elements $a, b, c \in A, \alpha, \beta, \gamma \in B$, and $x, y, z, w \in C$ :

$$
\begin{aligned}
\beth^{0}(f)(a) & =a f(1)-f(1) a, \\
\beth^{1}(f)\left((a) \otimes\left(\begin{array}{cc}
x & \alpha \\
& b
\end{array}\right)\right) & =a \varepsilon(\alpha \theta(x)) f(b)-f(a b \varepsilon(\alpha \theta(x)))+f(a) b \varepsilon(\alpha \theta(x)),
\end{aligned}
$$

and

$$
\begin{aligned}
\beth^{2}(f) & \left((a) \otimes\left(\begin{array}{ll}
x & \alpha \\
& b
\end{array}\right) \otimes\left(\begin{array}{ccc}
y & z & \beta \\
& w & \gamma \\
& & c
\end{array}\right)\right) \\
= & a \varepsilon(\alpha \beta \theta(x y z)) f\left((b) \otimes\left(\begin{array}{cc}
w & \gamma \\
& c
\end{array}\right)\right)-f\left((a b \varepsilon(\alpha \theta(x))) \otimes\left(\begin{array}{cc}
y z w & \beta \gamma \\
& c
\end{array}\right)\right) \\
& +f\left((a) \otimes\left(\begin{array}{cc}
x y & \alpha \beta \theta(z) \\
& b c \varepsilon(\gamma \theta(w))
\end{array}\right)\right)-f\left((a) \otimes\left(\begin{array}{cc}
x & \alpha \\
& b
\end{array}\right)\right) c \varepsilon(\beta \gamma \theta(y z w)) .
\end{aligned}
$$

Definition 2.4. ([2]) Let $\mathcal{Q}=(A, B, C, \varepsilon, \theta)$ be a quintuple. The cohomology of the complex $\mathbf{C}^{\bullet}(\mathcal{Q} ; M)$ is called the tertiary Hochschild cohomology of the quintuple $(A, B, C, \varepsilon, \theta)$ with coefficients in $M$, which is denoted by $\mathrm{H}^{*}((A, B, C, \varepsilon, \theta) ; M)$.

Example 2.5. ([2]) When $C=\mathbb{k}$, one recovers the secondary Hochschild cohomology $\mathrm{H}^{*}((A, B, \varepsilon) ; M)$, introduced in [8]. When $C=B=\mathbb{k}$, one recovers the usual Hochschild cohomology $\mathrm{H}^{*}(A, M)$, as in [6].

Example 2.6. In low dimensions, one can see $\mathrm{H}^{0}((A, B, C, \varepsilon, \theta) ; M)=\mathrm{H}^{0}((A, B, \varepsilon) ; M)=$ $\mathrm{H}^{0}(A, M)=[M, A]$ and $\mathrm{H}^{1}((A, B, C, \varepsilon, \theta) ; M)=\frac{\operatorname{Der}_{B, C}(A, M)}{\operatorname{Inn}_{\mathrm{k}}(A, M)}$. Here $\operatorname{Der}_{B, C}(A, M)$ denotes the module of all derivations of $A$ in $M$ which are both $B$-linear and $C$-linear, and $\operatorname{Inn}_{\mathbb{k}}(A, M)$ denotes the inner derivations.

\section{Higher order Hochschild COHOMOLOGY}

For this section we fix $A$ to be commutative. Consider a $\mathbb{k}_{k}[[t]]$-linear map $u: A[[t]] \longrightarrow$ $A[[t]]$ determined by

$$
u(a)=a+u_{1}(a) t+u_{2}(a) t^{2}+u_{3}(a) t^{3}+u_{4}(a) t^{4}+\cdots
$$

where each $u_{i}: A \longrightarrow A$ is $\mathbb{k}$-linear.

We note that (3.1) was investigated in [3] and an associativity-like condition for three elements was shown to be controlled by $\mathrm{H}_{S^{2}}^{*}(A, A)$. Here, we focus on $\mathrm{H}_{S^{3}}^{*}(A, A)$, and then ultimately generalize to $\mathrm{H}_{S^{d}}^{*}(A, A)$ for any $d \geq 1$. 
3.1. Modeling the 3-sphere. We start by recalling the complex associated to $\mathrm{H}_{S^{3}}^{*}(A, A)$. We get

$$
0 \rightarrow A \stackrel{\delta_{0}}{\rightarrow} A \stackrel{\delta_{1}}{\rightarrow} A \stackrel{\delta_{2}}{\rightarrow} \operatorname{Hom}(A, A) \stackrel{\delta_{3}}{\longrightarrow} \operatorname{Hom}\left(A^{\otimes 4}, A\right) \stackrel{\delta_{4}}{\rightarrow} \ldots
$$

by taking $d=3$ in (2.1) with $M=A$. Just as in (2.2), we want to focus on the map $\delta_{3}: \operatorname{Hom}(A, A) \longrightarrow \operatorname{Hom}\left(A^{\otimes 4}, A\right)$. For any $\mathbb{k}$-linear map $f: A \longrightarrow A$, we have that

$$
\delta_{3}(f)(a \otimes b \otimes c \otimes d)=a b c f(d)-a b f(c d)+a f(b c) d-f(a b) c d+f(a) b c d .
$$

Next we consider two $\mathbb{k}$-linear maps $f, g: A \longrightarrow A$. We define $f \circ g: A^{\otimes 4} \longrightarrow A$ by

$$
(f \circ g)(a \otimes b \otimes c \otimes d)=f(a b) g(c d)-a f(b c) g(d)-f(a) b c g(d)-f(a) g(b c) d .
$$

Furthermore, for three $\mathbb{k}$-linear maps $f, g, h: A \longrightarrow A$, we define $f \star g \star h: A^{\otimes 4} \longrightarrow A$ by

$$
(f \star g \star h)(a \otimes b \otimes c \otimes d)=-f(a) g(b c) h(d) .
$$

Suppose we desire the map $u$ from (3.1) to satisfy the property

$$
u(a b) u(c d)=u(a) u(b c) u(d) .
$$

Proposition 3.1. Let $u: A[[t]] \longrightarrow A[[t]]$ be defined as in (3.1).

(i) If $u$ satisfies (3.2) $\bmod t^{2}$, then $u_{1} \in Z_{S^{3}}^{3}(A, A)$.

(ii) If $u$ satisfies (3.2) $\bmod t^{n+1}$, then $u$ can be extended so that it satisfies (3.2) $\bmod t^{n+2}$ if and only if

$$
\sum_{i+j=n+1} u_{i} \circ u_{j}+\sum_{i+j+k=n+1} u_{i} \star u_{j} \star u_{k}=0 \in \mathrm{H}_{S^{3}}^{4}(A, A) .
$$

Proof. First observe that satisfying (3.2) yields

$$
\begin{aligned}
& \left(a b+u_{1}(a b) t+u_{2}(a b) t^{2}+u_{3}(a b) t^{3}+\cdots\right)\left(c d+u_{1}(c d) t+u_{2}(c d) t^{2}+u_{3}(c d) t^{3}+\cdots\right) \\
= & \left(a+u_{1}(a) t+u_{2}(a) t^{2}+\cdots\right)\left(b c+u_{1}(b c) t+u_{2}(b c) t^{2}+\cdots\right)\left(d+u_{1}(d) t+u_{2}(d) t^{2}+\cdots\right) .
\end{aligned}
$$

For $(i)$, we notice that in order to satisfy (3.2) $\bmod t^{2}$ we would need

$$
(a b)(c d)+a b u_{1}(c d) t+u_{1}(a b) c d t=a(b c) d+a b c u_{1}(d) t+a u_{1}(b c) d t+u_{1}(a) b c d t .
$$

This means

$$
a b c u_{1}(d)-a b u_{1}(c d)+a u_{1}(b c) d-u_{1}(a b) c d+u_{1}(a) b c d=0,
$$

and hence $\delta_{3}\left(u_{1}\right)(a \otimes b \otimes c \otimes d)=0$. Therefore $u_{1} \in \operatorname{Ker}\left(\delta_{3}\right)$ and so $u_{1}$ is a 3-cocycle. Thus $u_{1} \in Z_{S^{3}}^{3}(A, A)$.

For $(i i)$, we will show the cases in low dimension with the extension following naturally. For $n=1$, if (3.2) is satisfied $\bmod t^{2}$, and we desire equality $\bmod t^{3}$, then it reduces to

$$
\begin{gathered}
a b u_{2}(c d)+u_{2}(a b) c d+u_{1}(a b) u_{1}(c d) \\
=a b c u_{2}(d)+a u_{2}(b c) d+u_{2}(a) b c d+a u_{1}(b c) u_{1}(d)+u_{1}(a) b c u_{1}(d)+u_{1}(a) u_{1}(b c) d .
\end{gathered}
$$

One arranges (3.3) to become

$$
\begin{gathered}
a b c u_{2}(d)-a b u_{2}(c d)+a u_{2}(b c) d-u_{2}(a b) c d+u_{2}(a) b c d \\
=u_{1}(a b) u_{1}(c d)-a u_{1}(b c) u_{1}(d)-u_{1}(a) b c u_{1}(d)-u_{1}(a) u_{1}(b c) d .
\end{gathered}
$$

Writing (3.4) in a nice way yields $\delta_{3}\left(u_{2}\right)(a \otimes b \otimes c \otimes d)=\left(u_{1} \circ u_{1}\right)(a \otimes b \otimes c \otimes d)$. Thus, $u_{1} \circ u_{1} \in \operatorname{Im}\left(\delta_{3}\right)$, and therefore $u_{1} \circ u_{1}=0 \in \mathrm{H}_{S^{3}}^{4}(A, A)$, which was what we wanted. 
Notice how the latter sum is suppressed in the case $n=1$. For $n=2$, if we suppose (3.2) is satisfied $\bmod t^{3}$, and we desire equality $\bmod t^{4}$, then it reduces to

$$
\begin{gathered}
a b u_{3}(c d)+u_{3}(a b) c d+u_{1}(a b) u_{2}(c d)+u_{2}(a b) u_{1}(c d) \\
=a b c u_{3}(d)+a u_{3}(b c) d+u_{3}(a) b c d+a u_{1}(b c) u_{2}(d)+u_{1}(a) b c u_{2}(d)+u_{1}(a) u_{2}(b c) d \\
+a u_{2}(b c) u_{1}(d)+u_{2}(a) b c u_{1}(d)+u_{2}(a) u_{1}(b c) d+u_{1}(a) u_{1}(b c) u_{1}(d) .
\end{gathered}
$$

Rewriting (3.5) yields

$$
\begin{gathered}
a b c u_{3}(d)-a b u_{3}(c d)+a u_{3}(b c) d-u_{3}(a b) c d+u_{3}(a) b c d \\
=u_{1}(a b) u_{2}(c d)-a u_{1}(b c) u_{2}(d)-u_{1}(a) b c u_{2}(d)-u_{1}(a) u_{2}(b c) d \\
+u_{2}(a b) u_{1}(c d)-a u_{2}(b c) u_{1}(d)-u_{2}(a) b c u_{1}(d)-u_{2}(a) u_{1}(b c) d-u_{1}(a) u_{1}(b c) u_{1}(d) .
\end{gathered}
$$

Notice that (3.6) is $\delta_{3}\left(u_{3}\right)(a \otimes b \otimes c \otimes d)=\left(u_{1} \circ u_{2}+u_{2} \circ u_{1}+u_{1} \star u_{1} \star u_{1}\right)(a \otimes b \otimes c \otimes d)$. Thus $u_{1} \circ u_{2}+u_{2} \circ u_{1}+u_{1} \star u_{1} \star u_{1} \in \operatorname{Im}\left(\delta_{3}\right)$, and therefore $u_{1} \circ u_{2}+u_{2} \circ u_{1}+u_{1} \star u_{1} \star u_{1}=0 \in \mathrm{H}_{S^{3}}^{4}(A, A)$, as desired.

One can continue this construction for any $n \geq 1$.

3.2. Generalization. Fix $d \geq 1$. For $\mathbb{k}$-linear maps $f_{1}, \ldots, f_{m}: A \longrightarrow A$, we define $f_{1} \circ \cdots \circ f_{m}: A^{\otimes d+1} \longrightarrow A$ in the natural way, where $2 \leq m \leq\left\lceil\frac{d+2}{2}\right\rceil$.

Suppose we desire the map $u$ from (3.1) to satisfy the property

$$
\begin{cases}u\left(a_{1} a_{2}\right) \cdots u\left(a_{d} a_{d+1}\right)=u\left(a_{1}\right) u\left(a_{2} a_{3}\right) \cdots u\left(a_{d-1} a_{d}\right) u\left(a_{d+1}\right) & \text { if } d \text { is odd, } \\ u\left(a_{1} a_{2}\right) \cdots u\left(a_{d-1} a_{d}\right) u\left(a_{d+1}\right)=u\left(a_{1}\right) u\left(a_{2} a_{3}\right) \cdots u\left(a_{d} a_{d+1}\right) & \text { if } d \text { is even. }\end{cases}
$$

Theorem 3.2. Fix $d \geq 1$. Let $u: A[[t]] \longrightarrow A[[t]]$ be defined as in (3.1).

(i) If $u$ satisfies (3.7) $\bmod t^{2}$, then $u_{1} \in Z_{S^{d}}^{d}(A, A)$.

(ii) If $u$ satisfies (3.7) $\bmod t^{n+1}$, then $u$ can be extended so that it satisfies (3.7) $\bmod t^{n+2}$ if and only if

$$
\sum_{m=2}^{\left\lceil\frac{d+2}{2}\right\rceil}\left(\sum_{i_{1}+\cdots+i_{m}=n+1} u_{i_{1}} \circ \cdots \circ u_{i_{m}}\right)=0 \in \mathrm{H}_{S^{d}}^{d+1}(A, A) .
$$

Proof. Follows from Definition 2.1, the map given in (2.2), and the property in (3.7).

Example 3.3. For $d=1$, recall that the higher order Hochschild cohomology recovers the usual Hochschild cohomology. Therefore, one can apply Theorem 3.2 if one desires the map $u$ from (3.1) to satisfy the property $u(a b)=u(a) u(b)$.

Example 3.4. The case for $d=2$ recovers precisely what was done in [3].

Remark 3.5. Taking $d=3$ in Section 3.2 reduces to what was established in Section 3.1.

Corollary 3.6. Fix $d \geq 1$. Let $u: A[[t]] \longrightarrow A[[t]]$ be defined as in (3.1). If u satisfies (3.7) mod $t^{2}$, then the class of $u_{1} \in \mathrm{H}_{S^{d}}^{d}(A, A)$ is determined by the isomorphism class of $u$.

Proof. First, we know by Theorem 3.2(i) that $u_{1}$ is a $d$-cocycle. Next we consider two maps: $u(a)=a+u_{1}(a) t+u_{2}(a) t^{2}+\cdots$ and $w(a)=a+w_{1}(a) t+w_{2}(a) t^{2}+\cdots$. Suppose that we have $f: A[[t]] \longrightarrow A[[t]]$ an isomorphism given by $f(a)=a+f_{1}(a) t+f_{2}(a) t^{2}+\cdots$ such that we desire

$$
w(f(a))=f(u(a)) .
$$

In other words, the diagram in Figure 1 commutes. If (3.8) is satisfied $\bmod t^{2}$, then we get 


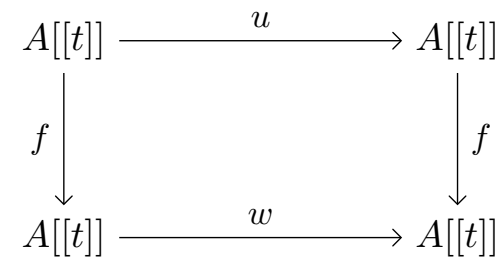

Figure 1. Commuting diagram

that $a+f_{1}(a) t+w_{1}(a) t=a+u_{1}(a) t+f_{1}(a) t$, and hence $\left(u_{1}-w_{1}\right)(a)=0$.

Using (2.3), we see that when $d$ is odd, we know that $\delta_{d-1}=0$, and when $d$ is even, we know that $\delta_{d-1}=$ id. Regardless, $u_{1}-w_{1} \in \operatorname{Im}\left(\delta_{d-1}\right)$. This shows that $u_{1}$ and $w_{1}$ are in the same class in $\mathrm{H}_{S^{d}}^{d}(A, A)$. The result follows.

Notice that all of the equalities contained in (3.7) are independent. Observe that $u(a b)=$ $u(a) u(b)$ (see Example 3.3) clearly implies the others, yet the converse fails. This is mainly because $u(1)$ need not equal 1 . The following result generalizes the implications.

Proposition 3.7. Let $u: A[[t]] \longrightarrow A[[t]]$ be defined as in (3.1). If u satisfies (3.7) for $d=n$ and for $d=m$, then $u$ satisfies (3.7) for $d=n+m$.

Proof. First suppose $n$ is odd and $m$ is even. This, of course, implies that $n+m$ is odd. We assume that $u$ satisfies the following:

$$
u\left(a_{1} a_{2}\right) \cdots u\left(a_{n} a_{n+1}\right)=u\left(a_{1}\right) u\left(a_{2} a_{3}\right) \cdots u\left(a_{n-1} a_{n}\right) u\left(a_{n+1}\right)
$$

and

$$
u\left(a_{1} a_{2}\right) \cdots u\left(a_{m-1} a_{m}\right) u\left(a_{m+1}\right)=u\left(a_{1}\right) u\left(a_{2} a_{3}\right) \cdots u\left(a_{m} a_{m+1}\right) .
$$

We want to show that $u$ satisfies (3.7) for $d=n+m$. We then observe that

$$
\begin{aligned}
& u\left(a_{1} a_{2}\right) \cdots u\left(a_{n} a_{n+1}\right) u\left(a_{n+2} a_{n+3}\right) \cdots u\left(a_{n+m} a_{n+m+1}\right) \\
& \quad=u\left(a_{1}\right) u\left(a_{2} a_{3}\right) \cdots u\left(a_{n-1} a_{n}\right) u\left(a_{n+1}\right) u\left(a_{n+2} a_{n+3}\right) \cdots u\left(a_{n+m} a_{n+m+1}\right) \text { by }(3.9) \\
& \quad=u\left(a_{1}\right) u\left(a_{2} a_{3}\right) \cdots u\left(a_{n-1} a_{n}\right) u\left(a_{n+1} a_{n+2}\right) \cdots u\left(a_{n+m-1} a_{n+m}\right) u\left(a_{n+m+1}\right) \text { by }(3.10),
\end{aligned}
$$

which was what we wanted. The cases for $n$ and $m$ both odd or both even can be done in a similar manner.

\section{Tertiary Hochschild COHOMOlogy}

In this section we impose nontrivial $B$-algebra and $C$-algebra structures on $A$ and establish a deformation theory corresponding to it. This is similar to what was done in [8].

First we let $\mathcal{Q}=(A, B, C, \varepsilon, \theta)$ be a quintuple. Note that here $A$ is not necessarily commutative. Since $\mathcal{Q}$ is a quintuple, notice that this induces a $B$-algebra structure on $A$ by way of the morphism $\varepsilon: B \longrightarrow A$, and it also induces a $C$-algebra structure on $A$ via the morphism $\varepsilon \circ \theta: C \longrightarrow A$ (see Definition 2.3).

Next for each $\alpha \in B$ and $x \in C$ we have a map $m_{\alpha}^{x}: A \otimes A \longrightarrow A$ given by $m_{\alpha}^{x}(a \otimes b)=$ $a b \varepsilon(\alpha) \varepsilon(\theta(x))$, where $a, b \in A$. One can verify that the following are easily satisfied for any 
$q \in \mathbb{k}, a, b, c \in A, \alpha, \beta, \gamma \in B$, and $x, y, z, w \in C:$

$$
\begin{gathered}
m_{\alpha+\beta}^{x}(a \otimes b)=m_{\alpha}^{x}(a \otimes b)+m_{\beta}^{x}(a \otimes b), \\
m_{\alpha}^{x+y}(a \otimes b)=m_{\alpha}^{x}(a \otimes b)+m_{\alpha}^{y}(a \otimes b), \\
m_{q \alpha}^{x}(a \otimes b)=q m_{\alpha}^{x}(a \otimes b)=m_{\alpha}^{q x}(a \otimes b), \\
m_{\alpha \beta \theta(z)}^{x y}\left(a \otimes m_{\gamma}^{w}(b \otimes c)\right)=m_{\beta \gamma}^{x y z}\left(m_{\alpha}^{x}(a \otimes b) \otimes c\right) .
\end{gathered}
$$

Conversely, now we suppose that $\theta: B \longrightarrow C$ is a morphism of commutative $\mathbb{k}_{k}$-algebras, and $A$ is a $\mathbb{k}$-vector space. Further suppose that we have a family of products $\mathcal{M}_{\mathcal{Q}}=\left\{m_{\alpha}^{x}\right.$ : $A \otimes A \longrightarrow A: \alpha \in B$ and $x \in C\}$ such that $\mathcal{M}_{\mathcal{Q}}$ satisfies the conditions in (4.1). Finally, suppose that $\left(A, m_{1}^{1}\right)$ is a $\mathbb{k}$-algebra with the identity element $1_{A} \in A$.

One can see that $\varepsilon_{\mathcal{M}_{\mathcal{Q}}}: B \longrightarrow A$ given by $\varepsilon_{\mathcal{M}_{\mathcal{Q}}}(\alpha)=m_{\alpha}^{1}\left(1_{A} \otimes 1_{A}\right)$ and $\varepsilon_{\mathcal{M}_{\mathcal{Q}}} \circ \theta: C \longrightarrow A$ given by $\left(\varepsilon_{\mathcal{M}_{\mathcal{Q}}} \circ \theta\right)(x)=m_{1}^{x}\left(1_{A} \otimes 1_{A}\right)$ are both morphisms of $\mathbb{k}$-algebras such that $\varepsilon_{\mathcal{M}_{\mathcal{Q}}}(B) \subseteq$ $\mathcal{Z}(A)$ and $\left(\varepsilon_{\mathcal{M}_{\mathcal{Q}}} \circ \theta\right)(C) \subseteq \mathcal{Z}(A)$, respectively. In particular, both of these maps respect sums, scalars, products, and the identity. As consequence, one has the following:

Proposition 4.1. Consider a morphism $\theta: B \longrightarrow C$ of commutative $\mathbb{k}$-algebras and a family of products $\mathcal{M}_{\mathcal{Q}}=\left\{m_{\alpha}^{x}: A \otimes A \longrightarrow A: \alpha \in B\right.$ and $\left.x \in C\right\}$ such that $\left(A, m_{1}^{1}\right)$ is a $\mathbb{k}$-algebra with unit $1_{A} \in A$. Then (4.1) holds if and only if $\varepsilon_{\mathcal{M}_{\mathcal{Q}}}: B \longrightarrow A$ given by $\varepsilon_{\mathcal{M}_{\mathcal{Q}}}(\alpha)=m_{\alpha}^{1}\left(1_{A} \otimes 1_{A}\right)$ and $\varepsilon_{\mathcal{M}_{\mathcal{Q}}} \circ \theta: C \longrightarrow A$ given by $\left(\varepsilon_{\mathcal{M}_{\mathcal{Q}}} \circ \theta\right)(x)=m_{1}^{x}\left(1_{A} \otimes 1_{A}\right)$ give a $B$-algebra and $C$-algebra structure on $A$, respectively.

Proof. Follows from the above discussion.

4.1. A deformation theory. Let $\mathcal{Q}=(A, B, C, \varepsilon, \theta)$ be a quintuple. Suppose that for each $i \geq 1$ we have a $\mathbb{k}$-linear map $c_{i}: A^{\otimes 2} \otimes B \otimes C \longrightarrow A$. For each $\alpha \in B$ and $x \in C$, we define $m_{\alpha, t}^{x}: A[[t]] \otimes A[[t]] \longrightarrow A[[t]]$ determined by

$$
m_{\alpha, t}^{x}(a \otimes b)=a b \varepsilon(\alpha \theta(x))+c_{1}\left((a) \otimes\left(\begin{array}{cc}
x & \alpha \\
& b
\end{array}\right)\right) t+c_{2}\left((a) \otimes\left(\begin{array}{cc}
x & \alpha \\
& b
\end{array}\right)\right) t^{2}+\cdots
$$

where $a, b \in A$. Suppose we desire the family of products $\mathcal{M}_{\mathcal{Q}}=\left\{m_{\alpha, t}^{x}: \alpha \in B\right.$ and $\left.x \in C\right\}$ to satisfy the following associativity condition:

$$
m_{\alpha \beta \theta(z), t}^{x y}\left(a \otimes m_{\gamma, t}^{w}(b \otimes c)\right)=m_{\beta \gamma, t}^{y z w}\left(m_{\alpha, t}^{x}(a \otimes b) \otimes c\right),
$$

where $a, b, c \in A, \alpha, \beta, \gamma \in B$, and $x, y, z, w \in C$.

Remark 4.2. Taking $C=\mathbb{k}$, one recovers the family of products $\mathcal{M}$ discussed in [8], whereas taking $C=B=\mathbb{k}$ reduces to the usual product $m_{t}$ studied in [4].

For $\mathbb{k}$-linear maps $f, g: A^{\otimes 2} \otimes B \otimes C \longrightarrow A$, we define $f \circ g: A^{\otimes 3} \otimes B^{\otimes 3} \otimes C^{\otimes 4} \longrightarrow A$ by the following:

$$
\begin{aligned}
& (f \circ g)\left((a) \otimes\left(\begin{array}{cc}
x & \alpha \\
& b
\end{array}\right) \otimes\left(\begin{array}{ccc}
y & z & \beta \\
& w & \gamma \\
& & c
\end{array}\right)\right)
\end{aligned}
$$

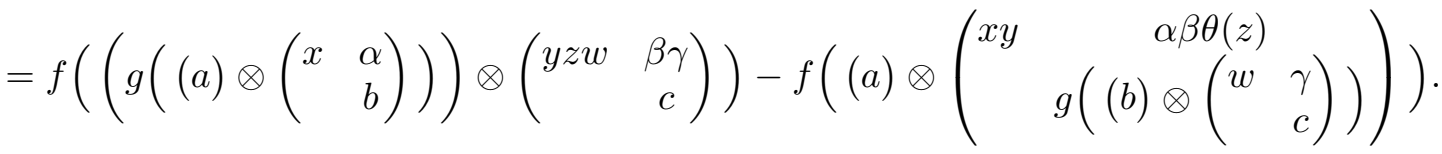

Theorem 4.3. Let $\mathcal{Q}=(A, B, C, \varepsilon, \theta)$ be a quintuple. Suppose $\mathcal{M}_{\mathcal{Q}}=\left\{m_{\alpha, t}^{x} \quad: \quad \alpha \in\right.$ $B$ and $x \in C\}$ is the family of products defined as in (4.2). 
(i) If the family of products $\mathcal{M}_{\mathcal{Q}}$ satisfies (4.3) $\bmod t^{2}$, then $c_{1} \in Z^{2}((A, B, C, \varepsilon, \theta) ; A)$.

(ii) If the family of products $\mathcal{M}_{\mathcal{Q}}$ satisfies $(4.3) \bmod t^{n+1}$, then $\mathcal{M}_{\mathcal{Q}}$ can be extended so that the family of products satisfies (4.3) $\bmod t^{n+2}$ if and only if

$$
\sum_{i+j=n+1} c_{i} \circ c_{j}=0 \in \mathrm{H}^{3}((A, B, C, \varepsilon, \theta) ; A) .
$$

Proof. For $(i)$, in order to satisfy $(4.3) \bmod t^{2}$ we need

$$
\begin{aligned}
& c_{1}\left((a) \otimes\left(\begin{array}{cc}
x y & \alpha \beta \theta(z) \\
& b c \varepsilon(\gamma \theta(w))
\end{array}\right)\right)+a \varepsilon(\alpha \beta \theta(x y z)) c_{1}\left((b) \otimes\left(\begin{array}{ll}
w & \gamma \\
& c
\end{array}\right)\right) \\
= & c_{1}\left((a b \varepsilon(\alpha \theta(x))) \otimes\left(\begin{array}{cc}
y z w & \beta \gamma \\
& c
\end{array}\right)\right)+c_{1}\left((a) \otimes\left(\begin{array}{cc}
x & \alpha \\
& b
\end{array}\right)\right) c \varepsilon(\beta \gamma \theta(y z w))
\end{aligned}
$$

which can be rearranged as

$$
\begin{gathered}
a \varepsilon(\alpha \beta \theta(x y z)) c_{1}\left((b) \otimes\left(\begin{array}{ll}
w & \gamma \\
& c
\end{array}\right)\right)-c_{1}\left((a b \varepsilon(\alpha \theta(x))) \otimes\left(\begin{array}{cc}
y z w & \beta \gamma \\
c
\end{array}\right)\right) \\
+c_{1}\left((a) \otimes\left(\begin{array}{cc}
x y & \alpha \beta \theta(z) \\
& b c \varepsilon(\gamma \theta(w))
\end{array}\right)\right)-c_{1}\left((a) \otimes\left(\begin{array}{cc}
x & \alpha \\
& b
\end{array}\right)\right) c \varepsilon(\beta \gamma \theta(y z w))=0 .
\end{gathered}
$$

Hence $\beth^{2}\left(c_{1}\right)=0$, and so $c_{1} \in \operatorname{Ker}\left(\beth^{2}\right)$. This implies that $c_{1}$ is a 2-cocycle, and thus $c_{1} \in Z^{2}((A, B, C, \varepsilon, \theta) ; A)$.

For $(i i)$, we consider the case $n=1$. Assuming the family of products $\mathcal{M}_{\mathcal{Q}}$ satisfies (4.3) $\bmod t^{2}$, if we further desire $\mathcal{M}_{\mathcal{Q}}$ to satisfy (4.3) $\bmod t^{3}$, we then get that

$$
\begin{gathered}
c_{2}\left((a) \otimes\left(\begin{array}{cc}
x y & \alpha \beta \theta(z) \\
& b c \varepsilon(\gamma \theta(w))
\end{array}\right)\right)+c_{1}\left((a) \otimes\left(\begin{array}{cc}
x y & \alpha \beta \theta(z) \\
& c_{1}\left((b) \otimes\left(\begin{array}{ll}
w & \gamma \\
& c
\end{array}\right)\right)
\end{array}\right)\right) \\
+a \varepsilon(\alpha \beta \theta(x y z)) c_{2}\left((b) \otimes\left(\begin{array}{cc}
w & \gamma \\
& c
\end{array}\right)\right)=c_{2}\left((a b \varepsilon(\alpha \theta(x))) \otimes\left(\begin{array}{cc}
y z w & \beta \gamma \\
c
\end{array}\right)\right) \\
+c_{1}\left(\left(c_{1}\left((a) \otimes\left(\begin{array}{cc}
x & \alpha \\
& b
\end{array}\right)\right)\right) \otimes\left(\begin{array}{cc}
y z w & \beta \gamma \\
& c
\end{array}\right)\right)+c_{2}\left((a) \otimes\left(\begin{array}{cc}
x & \alpha \\
b
\end{array}\right)\right) c \varepsilon(\beta \gamma \theta(y z w)) .
\end{gathered}
$$

Notice that (4.4) can be rewritten as $\beth^{2}\left(c_{2}\right)=c_{1} \circ c_{1}$, which describes the obstruction. Hence since $c_{1} \circ c_{1} \in \operatorname{Im}\left(\beth^{2}\right)$, then $c_{1} \circ c_{1}=0 \in \mathrm{H}^{3}((A, B, C, \varepsilon, \theta) ; A)$. Observe that one can do this for any $n \geq 1$ in order to extend associativity of the family of products $\mathcal{M}_{\mathcal{Q}}$, as is traditional for these types of deformation arguments.

Remark 4.4. As one would expect based on Example 2.5 and Remark 4.2, we see that Theorem 4.3 reduces to the known deformation theory results corresponding to the secondary and usual Hochschild cohomologies found in [8] and [4] when one takes $C=\mathbb{k}$ and $C=B=\mathbb{k}$, respectively.

Corollary 4.5. Let $\mathcal{Q}=(A, B, C, \varepsilon, \theta)$ be a quintuple. Suppose $\mathcal{M}_{\mathcal{Q}}=\left\{m_{\alpha, t}^{x}: \quad \alpha \in\right.$ $B$ and $x \in C\}$ is the family of products defined as in (4.2). If the family of products $\mathcal{M}_{\mathcal{Q}}$ satisfies (4.3) mod $t^{2}$, then the class of $c_{1} \in \mathrm{H}^{2}((A, B, C, \varepsilon, \theta) ; A)$ is determined by the isomorphism class of $\mathcal{M}_{\mathcal{Q}}$. 
Proof. First, we know by Theorem 4.3(i) that $c_{1}$ is a 2-cocycle. Next we consider two families of products $\left\{m_{\alpha, t}^{x}: \alpha \in B\right.$ and $\left.x \in C\right\}$ and $\left\{p_{\alpha, t}^{x}: \alpha \in B\right.$ and $\left.x \in C\right\}$ :

$$
m_{\alpha, t}^{x}(a \otimes b)=a b \varepsilon(\alpha \theta(x))+c_{1}\left((a) \otimes\left(\begin{array}{cc}
x & \alpha \\
& b
\end{array}\right)\right) t+c_{2}\left((a) \otimes\left(\begin{array}{cc}
x & \alpha \\
& b
\end{array}\right)\right) t^{2}+\cdots
$$

and

$$
p_{\alpha, t}^{x}(a \otimes b)=a b \varepsilon(\alpha \theta(x))+d_{1}\left((a) \otimes\left(\begin{array}{cc}
x & \alpha \\
& b
\end{array}\right)\right) t+d_{2}\left((a) \otimes\left(\begin{array}{cc}
x & \alpha \\
& b
\end{array}\right)\right) t^{2}+\cdots .
$$

Suppose $f: A[[t]] \longrightarrow A[[t]]$ is an isomorphism given by $f(a)=a+f_{1}(a) t+f_{2}(a) t^{2}+\cdots$ such that we desire

$$
p_{\alpha, t}^{x}(f(a) \otimes f(b))=f\left(m_{\alpha, t}^{x}(a \otimes b)\right) .
$$

Equivalently, the diagram in Figure 2 commutes. If (4.5) is satisfied $\bmod t^{2}$, then we get

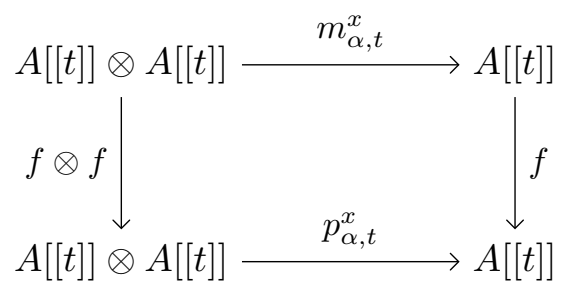

Figure 2. Commuting diagram

$$
\begin{gathered}
a b \varepsilon(\alpha \theta(x))+d_{1}\left((a) \otimes\left(\begin{array}{ll}
x & \alpha \\
& b
\end{array}\right)\right) t+a \varepsilon(\alpha \theta(x)) f_{1}(b) t+f_{1}(a) b \varepsilon(\alpha \theta(x)) t \\
=a b \varepsilon(\alpha \theta(x))+f_{1}(a b \varepsilon(\alpha \theta(x))) t+c_{1}\left((a) \otimes\left(\begin{array}{cc}
x & \alpha \\
& b
\end{array}\right)\right) t .
\end{gathered}
$$

Rearranging (4.6) yields

$$
\begin{aligned}
& a \varepsilon(\alpha \theta(x)) f_{1}(b)-f_{1}(a b \varepsilon(\alpha \theta(x)))+f_{1}(a) b \varepsilon(\alpha \theta(x)) \\
& \quad=c_{1}\left((a) \otimes\left(\begin{array}{cc}
x & \alpha \\
& b
\end{array}\right)\right)-d_{1}\left((a) \otimes\left(\begin{array}{cc}
x & \alpha \\
& b
\end{array}\right)\right) .
\end{aligned}
$$

One can then rewrite (4.7) as $c_{1}-d_{1}=\beth^{1}\left(f_{1}\right)$. This shows that $c_{1}-d_{1} \in \operatorname{Im}\left(\beth^{1}\right)$ and hence $c_{1}$ and $d_{1}$ are in the same class in $\mathrm{H}^{2}((A, B, C, \varepsilon, \theta) ; A)$.

4.2. Extensions and remarks. One observes that the tertiary Hochschild cohomology controls a deformation theory on $A[[t]]$ that has both nontrivial $B$-algebra and $C$-algebra structures. However, as mentioned in [2], there is nothing special about the tertiary Hochschild cohomology. As one could imagine, one can extend to a so-called quaternary Hochschild cohomology to investigate deformations of $A[[t]]$ that have three additional algebra structures (coming from $B, C$, and $D$, say). In short, however finitely many distinct nontrivial algebra structures that one desires to impose on $A[[t]]$, one can conceivably devise the appropriate generalized Hochschild cohomology that will control that deformation theory. 


\section{REFERENCES}

[1] D. W. Anderson. Chain functors and homology theories. Symposium on Algebraic Topology (Battelle Seattle Res. Center, Seattle, Wash., 1971), pages 1-12. Lecture notes in Math., Vol. 249, 1971.

[2] Samuel Carolus and Jacob Laubacher. Simplicial structures over the 3-sphere and generalized higher order Hochschild homology. arXiv:1707.03863, 2017.

[3] Samuel Carolus and Mihai D. Staic. G-algebra structure on the higher order Hochschild cohomology $H_{S^{2}}^{*}(A, A)$. arXiv:1804.05096, 2018.

[4] Murray Gerstenhaber. On the deformation of rings and algebras. Ann. of Math. (2), 79:59-103, 1964.

[5] Grégory Ginot. Higher order Hochschild cohomology. C. R. Math. Acad. Sci. Paris, 346(1-2):5-10, 2008.

[6] Gerhard Hochschild. On the cohomology groups of an associative algebra. Ann. of Math. (2), 46:58-67, 1945.

[7] Teimuraz Pirashvili. Hodge decomposition for higher order Hochschild homology. Ann. Sci. École Norm. Sup. (4), 33(2):151-179, 2000.

[8] Mihai D. Staic. Secondary Hochschild cohomology. Algebr. Represent. Theory, 19(1):47-56, 2016.

Department of Mathematics and Statistics, Ohio Northern University, Ada, Ohio 45810

E-mail address: s-carolus@onu.edu

Department of Mathematics, St. Norbert College, De Pere, Wisconsin 54115

E-mail address: samuel.hokamp@snc.edu

Department of Mathematics, St. Norbert College, De Pere, Wisconsin 54115

E-mail address: jacob.laubacher@snc.edu 